\title{
Synthesis of planar chiral cobalt metallocenes by microwave-assisted diastereoselective complexation
}

Caroline J. Taylor, Majid Motevalli and Christopher J. Richards*

School of Biological and Chemical Sciences, Queen Mary, University of London, Mile End Road, E1 4NS, UK.

Fax: +44(0)20 7882 7427; Tel: +44(0)2078823271

\section{Supporting Information}

\section{Index}

General Procedure for the preparation of Propargyl alcohols $\mathbf{4 a}$ and $\mathbf{4 b}$

General procedure for the preparation of ethers $\mathbf{5 a}$ and $\mathbf{5 b}$

General procedure for the Sonogashira reaction of ethers $\mathbf{5 a}$ and $\mathbf{5 b}$

to give ethers $\mathbf{6 a}$ and $\mathbf{6 b}$

General procedure for the preparation of esters $7 \mathbf{a}$ and $7 \mathbf{b} \quad$ S5

General procedure for the Sonogashira reaction of $7 \mathbf{a}$ and $7 \mathbf{b}$ to give $\mathbf{8 a}$ and $\mathbf{8 b} \quad$ S6

$\begin{array}{ll}\text { Synthesis of }(S)-\mathbf{4 b} & \text { S6 }\end{array}$

Synthesis of $(S)-\mathbf{8 b}$ and subsequent reaction with $\mathrm{CpCo}(\mathrm{CO})_{2} \quad \mathrm{~S} 8$

X-Ray crystallography - Compound 9a $\quad$ S9

$\begin{array}{ll}\text { Representation of 9a with numbering scheme } & \text { S10 }\end{array}$

$\begin{array}{ll}\text { X-Ray crystallography - Compound 11a } & \text { S11 }\end{array}$

$\begin{array}{lr}\text { Representation of 11a with numbering scheme } & \text { S12 }\end{array}$

$\begin{array}{ll}\text { References } & \text { S13 }\end{array}$ 
General considerations. Petroleum ether refers to that fraction boiling in the range $40-60{ }^{\circ} \mathrm{C}$. THF was distilled from sodium benzophenone ketyl, and toluene from sodium wire. Silica gel 40-63 $\mu \mathrm{m}$ was used for chromatography. Butyllithium was used as a $2.5 \mathrm{M}$ solution in hexanes. Microwave reactions were carried with a CEM Discover (300 W). Thin layer chromatography was performed on silica gel $60 \mathrm{~F}_{254}$ plates (Merck).

\section{General procedure for the preparation of propargyl alcohols $4 a$ and $4 b$}

To a stirred solution of phenylacetylene $(3.0 \mathrm{~mL}, 27 \mathrm{mmol})$ in THF $(20 \mathrm{~mL})$ under a nitrogen atmosphere at $c a .-80{ }^{\circ} \mathrm{C}$ was added $n$-BuLi (2.5 M in Hexanes) $(12.96 \mathrm{~mL}, 32.4 \mathrm{mmol})$. The resulting mixture was allowed to warm to room temperature over $3 \mathrm{~h}$ during which time the colour of the solution changed from pale yellow to dark brown/green. The mixture was then recooled to $c a .-80{ }^{\circ} \mathrm{C}$ and either acetaldehyde or isobutyraldehyde $(27 \mathrm{mmol})$ was added, and the mixture instantly changed colour from brown to yellow. The reaction mixture was allowed to warm to room temperature and was then stirred for $72 \mathrm{~h}$. Addition of water $(20 \mathrm{~mL})$ was followed by extraction with ether $(2 \times 20 \mathrm{~mL})$, drying of the organic layer $\left(\mathrm{MgSO}_{4}\right)$ and removal of the solvent in vacuo. The products were purified by bulb to bulb distillation.

4a: Product isolated as a colourless oil (3.92 g, $98 \%) .{ }^{1} \mathrm{H}$ NMR $\left(\delta, 270 \mathrm{MHz}, \mathrm{CDCl}_{3}\right) 1.54(3$ H, d, J 6.4), 3.42 (1 H, brs), 4.76 (1 H, q, J 6.7), 7.25 - $7.27(3 \mathrm{H}, \mathrm{m}), 7.39$ - $7.51(2 \mathrm{H}, \mathrm{m})$; ${ }^{13} \mathrm{C}$ NMR $\left(\delta, 67 \mathrm{MHz}, \mathrm{CDCl}_{3}\right)$ 24.5, 58.7, 83.9, 91.5, 122.9, 128.4, 131.8; high-resolution MS ( $m / z$, EI) found $\mathrm{MNH}_{4}{ }^{+} 164.1068 ; \mathrm{C}_{10} \mathrm{H}_{14} \mathrm{NO}$ requires 164.1070 .

4b: Product isolated as a colourless oil (4.38 g, $92 \%) .{ }^{1} \mathrm{H}$ NMR $\left(\delta, 270 \mathrm{MHz}, \mathrm{CDCl}_{3}\right) 1.05(3$ H, d, J 6.9), 1.11 (3 H, d, J 7.0), 1.98 (1 H, octet, $J$ 6.7), $3.03(1 \mathrm{H}, \mathrm{d}, J 5.4), 4.38(1 \mathrm{H}, \mathrm{t}, J$ 5.7), 7.21 - 7.29 (3 H, m), $7.43-7.44(2 \mathrm{H}, \mathrm{m}) ;{ }^{13} \mathrm{C} \mathrm{NMR}\left(\delta, 67 \mathrm{MHz}, \mathrm{CDCl}_{3}\right)$ 17.7, 18.4, 
$34.8,68.3,85.5,89.3,123.0,128.3,131.8$; high-resolution $\mathrm{MS}(\mathrm{m} / z, \mathrm{EI})$ found $\mathrm{MNH}_{4}^{+}$ 192.1381; $\mathrm{C}_{12} \mathrm{H}_{18} \mathrm{NO}$ requires 192.1383 .

\section{General procedure for the preparation of ethers $5 \mathrm{a}$ and $5 \mathrm{~b}$}

Either alcohol $\mathbf{4 a}$ or $\mathbf{4 b}$ (1 eq), propargyl bromide (1.2 eq) and 18-crown-6 (0.5 eq) were stirred vigorously with saturated aqueous $\mathrm{KOH}(20 \mathrm{~mL} / \mathrm{mmol}$ of $\mathbf{4 a} / \mathbf{b})$ and the mixture heated at reflux for 48 hours. After cooling to room temperature the product was extracted into dichloromethane $(2 \times 20 \mathrm{~mL})$, the organic layers were dried $\left(\mathrm{MgSO}_{4}\right)$, and the solvent removed in vacuo. The product was purified by column chromatography $(30 \%$ EtOAc/petroleum ether).

5a: 4a $(0.200 \mathrm{~g}, 1.3 \mathrm{mmol})$ gave 5a as a pale yellow oil after purification $(0.160 \mathrm{~g}, 64 \%) .{ }^{1} \mathrm{H}$ NMR $\left(\delta, 270 \mathrm{MHz}, \mathrm{CDCl}_{3}\right) 1.56(3 \mathrm{H}, \mathrm{d}, J 6.7), 2.46(1 \mathrm{H}, \mathrm{s}), 4.32(1 \mathrm{H}, \mathrm{d}, J 15), 4.40(1 \mathrm{H}$, d, $J$ 15) $4.64\left(1 \mathrm{H}, \mathrm{q}, J\right.$ 6.7), 7.22 - $7.31(3 \mathrm{H}, \mathrm{m}), 7.4-7.5(2 \mathrm{H}, \mathrm{m}) ;{ }^{13} \mathrm{C} \mathrm{NMR}(\delta, 67 \mathrm{MHz}$, $\left.\mathrm{CDCl}_{3}\right) 22.1,55.9,64.8,74.6,79.7,85.7,88.1,122.6,128.4,131.9 ; \mathrm{MS}(\mathrm{m} / z, \mathrm{EI}) 185\left(\mathrm{MH}^{+}\right.$, $15 \%)$.

5b: 4b (1.66g, $9.5 \mathrm{mmol})$ gave $5 \mathbf{b}$ as a yellow oil $(1.66 \mathrm{~g}, 82 \%) .{ }^{1} \mathrm{H}$ NMR $(\delta, 270 \mathrm{MHz}$, $\left.\mathrm{CDCl}_{3}\right) 1.09(3 \mathrm{H}, \mathrm{d}, J$ 6.4), $1.11(3 \mathrm{H}, \mathrm{d}, J 5.9), 2.06$ (1 H, octet, $J$ 6.7), 2.45 (1 H, t, $J 2.5)$, $4.33(1 \mathrm{H}, \mathrm{d}, J 5.7), 4.34(1 \mathrm{H}, \mathrm{d}, J 16.6,2.5), 4.43(1 \mathrm{H}, \mathrm{dd}, J 15.6,2.5), 7.30-7.33(3 \mathrm{H}, \mathrm{m})$, 7.44 - $7.52(2 \mathrm{H}, \mathrm{m}) ;{ }^{13} \mathrm{C} \mathrm{NMR}\left(\delta, 67 \mathrm{MHz}, \mathrm{CDCl}_{3}\right)$ 18.0, 18.7, 33.3, 56.1, 74.4, 74.5, 79.9, 86.3, 87.1, 122.8, 128.4, 128.5, 131.9; high-resolution MS $\left(\mathrm{m} / \mathrm{z}\right.$, EI) found $\mathrm{MNH}_{4}{ }^{+}$230.1542; $\mathrm{C}_{15} \mathrm{H}_{20} \mathrm{NO}$ requires 230.1539 . 
General procedure for the Sonogashira reaction of ethers $5 \mathrm{a}$ and $5 \mathrm{~b}$ to give ethers $6 \mathrm{a}$ and $6 b$

Either $\mathbf{5 a}$ or $\mathbf{5 b}(1 \mathrm{eq})$ were stirred in triethylamine $(10 \mathrm{~mL} / \mathrm{mmol} \mathbf{5 a} / \mathbf{b})$ and to this solution was added $\mathrm{CuI}(10 \mathrm{~mol} \%), \mathrm{PdCl}_{2}\left(\mathrm{PPh}_{3}\right)_{2}(3 \mathrm{~mol} \%)$, and iodobenzene $(1 \mathrm{eq})$ and the mixture was stirred overnight under nitrogen. The reaction mixture was poured into a mixture of dichloromethane $(20 \mathrm{~mL})$ and saturated aqueous ammonium chloride $(20 \mathrm{~mL})$, the organic layer was separated, dried $\left(\mathrm{MgSO}_{4}\right)$ and the solvent removed in vacuo. The products were purified by column chromatography (10\% EtOAc/petroleum ether).

6a: Use of 5a (5.28 g, $0.028 \mathrm{~mol})$ gave $6 \mathbf{a}$ as a yellow oil $\left(5.47 \mathrm{~g}, 73 \%\right.$ ). IR (thin film) $v_{\max }$ 3035, $1489 \mathrm{~cm}^{-1} ;{ }^{1} \mathrm{H}$ NMR $\left(\delta, 270 \mathrm{MHz}, \mathrm{CDCl}_{3}\right) 1.61(3 \mathrm{H}, \mathrm{d}, J 6.7), 4.51(1 \mathrm{H}, \mathrm{d}, J 15.6)$, $4.66(1 \mathrm{H}, \mathrm{d}, J 15.6), 4.72(1 \mathrm{H}, \mathrm{q}, J 6.7), 7.07-7.72(10 \mathrm{H}, \mathrm{m}) ;{ }^{13} \mathrm{C} \mathrm{NMR}(\delta, 67 \mathrm{MHz}$, $\left.\mathrm{CDCl}_{3}\right)$ 22.3, 56.8, 64.8, 85.1, 86.0, 86.4, 88.4, 122.7, 122.8, 127.6, 128.4, 128.6, 130.4, 131.7, 131.9, 132.0, 137.6; high-resolution MS (m/z, EI) found $\mathrm{MNH}_{4}{ }^{+} 278.1541 ; \mathrm{C}_{19} \mathrm{H}_{20} \mathrm{NO}$ requires 278.1359 .

6b: Use of 5b (1.60 g, $7.5 \mathrm{mmol})$ gave $\mathbf{6 b}$ as a yellow oil $(2.05 \mathrm{~g}, 94 \%)$. IR (thin film) $v_{\max }$ 2961, $1689 \mathrm{~cm}^{-1}$; ${ }^{1} \mathrm{H}$ NMR $\left(\delta, 270 \mathrm{MHz}, \mathrm{CDCl}_{3}\right) 1.13$ (3 H, d, $\left.J 6.4\right), 1.15(3 \mathrm{H}, \mathrm{d}, J 6.4), 2.12$ (1 H, octet, $J$ 6.4), 4.40 (1 H, d, $J$ 5.6), 4.57 (1 H, J 15.8), 4.67 (1 H, J 15.8), 7.31 - 7.74 (10 $\mathrm{H}, \mathrm{m}) ;{ }^{13} \mathrm{C} \mathrm{NMR}\left(\delta, 67 \mathrm{MHz}, \mathrm{CDCl}_{3}\right)$ 18.2, 18.9, 33.3, 57.0, 74.5, 85.4, 86.3, 86.6, 94.6, 122.9, 127.6, 128.4, 128.6, 130.4, 132.0, 137.6; high-resolution MS $\left(m / z\right.$, EI) found $\mathrm{MNH}_{4}^{+}$ 306.1856; $\mathrm{C}_{21} \mathrm{H}_{24} \mathrm{NO}$ requires 306.1852 . 


\section{General procedure for the preparation of esters $7 \mathbf{a}$ and $7 \mathrm{~b}$}

2-Iodobenzoic acid (1.2 eq) was refluxed in thionyl chloride $(10 \mathrm{~mL} / \mathrm{mmol})$ overnight. The excess thionyl chloride was removed in vacuo and the residue was dissolved in $\mathrm{CH}_{2} \mathrm{Cl}_{2}(10$ $20 \mathrm{~mL}$ ) and cooled to $0{ }^{\circ} \mathrm{C}$. To the cooled solution was added either alcohol $\mathbf{4 a}$ or $\mathbf{4 b}(1 \mathrm{eq})$ and triethylamine (1.3 eq). This mixture was stirred for $4 \mathrm{~h}$ and allowed to warm to room temperature before being poured into $\mathrm{H}_{2} \mathrm{O}(20 \mathrm{~mL})$ and $\mathrm{CH}_{2} \mathrm{Cl}_{2}(30 \mathrm{~mL})$. The organic layer was separated, dried $\left(\mathrm{MgSO}_{4}\right)$ and the solvent removed in vacuo. The products were purified by column chromatography (30\% EtOAc/petroleum ether).

7a: Use of $4 \mathbf{a}(7.4 \mathrm{~g}, 50 \mathrm{mmol})$ gave $7 \mathbf{a}$ as a yellow oil $(10.89 \mathrm{~g}, 28 \mathrm{mmol}, 57 \%)$. IR (thin film) $v_{\max } 2986,1732 \mathrm{~cm}^{-1} ;{ }^{1} \mathrm{H}$ NMR $\left(\delta, 270 \mathrm{MHz}, \mathrm{CDCl}_{3}\right) 1.24(3 \mathrm{H}, \mathrm{d}, J 6.4), 5.53(1 \mathrm{H}, \mathrm{q}, J$ 7.1), 6.37 (1 H, t, J 7.6), 6.75 - 6.82 (4 H, m), 6.96 - 6.97 (2 H, m), 7.27 (1 H, d, J 7.7), 7.37 $\left(1 \mathrm{H}, \mathrm{d}, J\right.$ 7.7); 13C NMR $\left(\delta, 67 \mathrm{MHz}, \mathrm{CDCl}_{3}\right)$ 21.4, 62.0, 85.0, 87.5, 94.1, 121.9, 127.8, 128.2, 128.6, 130.7, 131.6, 131.9, 132.6, 134.6, 140.0, 164.7; high-resolution MS $(\mathrm{m} / \mathrm{z}$, EI) found $\mathrm{MNH}_{4}{ }^{+}$394.0298; $\mathrm{C}_{17} \mathrm{H}_{17} \mathrm{INO}_{2}$ requires 394.0298.

7b: Use of $\mathbf{4 b}(5.82 \mathrm{~g}, 30 \mathrm{mmol})$ gave $7 \mathbf{b}$ as a yellow oil $(8.48 \mathrm{~g}, 63 \%)$. IR (thin film) $v_{\max }$ 2965, $1730 \mathrm{~cm}^{-1} ;{ }^{1} \mathrm{H}$ NMR $\left(\delta, 270 \mathrm{MHz}, \mathrm{CDCl}_{3}\right) 0.62(3 \mathrm{H}, \mathrm{d}, J 6.7), 0.67(3 \mathrm{H}, \mathrm{d}, J$ 6.4), 1.62 - $1.81(1 \mathrm{H}, \mathrm{m}), 5.26(1 \mathrm{H}, \mathrm{d}, J$ 5.2), 6.50 - $6.55(1 \mathrm{H}, \mathrm{m}), 6.72-6.91(6 \mathrm{H}, \mathrm{m}), 7.27$ - 7.37 (2 $\mathrm{H}, \mathrm{m}) ;{ }^{13} \mathrm{C} \mathrm{NMR}\left(\delta, 67 \mathrm{MHz}, \mathrm{CDCl}_{3}\right)$ 17.8, 18.3, 32.5, 70.3, 85.2, 86.3, 94.2, 122.0, 127.8, $128.2,128.5,130.7,131.5,132.6,134.6,141.1,141.5,164.6$; high-resolution MS $(\mathrm{m} / z$, EI) found $\mathrm{MNH}_{4}{ }^{+}$422.0612; $\mathrm{C}_{19} \mathrm{H}_{21} \mathrm{INO}_{2}$ requires 422.0611 . 


\section{General procedure for the Sonogashira reaction of $7 a$ and $7 b$ to give $8 a$ and $8 b$}

Ester $7 \mathbf{a}$ or $7 \mathbf{b}(1 \mathrm{eq})$ were stirred in triethylamine $(10 \mathrm{~mL} / \mathrm{mmol} \mathbf{7 a} / \mathbf{b})$ to this solution was added $\mathrm{CuI}(10 \mathrm{~mol} \%), \mathrm{PdCl}_{2}\left(\mathrm{PPh}_{3}\right)_{2}(3 \mathrm{~mol} \%)$, and phenyl acetylene $(1 \mathrm{eq})$, and the mixture was stirred for $24 \mathrm{~h}$ under nitrogen at $60{ }^{\circ} \mathrm{C}$. The reaction mixture was poured into a mixture of dichloromethane $(20 \mathrm{~mL})$ and saturated aqueous ammonium chloride $(10 \mathrm{~mL})$, the organic layer was separated, dried $\left(\mathrm{MgSO}_{4}\right)$, and the solvent removed in vacuo. The products were purified by column chromatography (30\% EtOAc/petroleum ether).

8a: Use of 7a (1.89 g, $4.94 \mathrm{mmol})$ gave 8a the product as a brown oil (1.72 g, $98 \%$ ). IR (thin film) $v_{\max } 1727 \mathrm{~cm}^{-1} ;{ }^{1} \mathrm{H}$ NMR $\left(\delta, 270 \mathrm{MHz}, \mathrm{CDCl}_{3}\right) 1.72(3 \mathrm{H}, \mathrm{d}, J 6.7), 6.03(1 \mathrm{H}, \mathrm{q}, J 6.7)$, 7.10 (1 H, td, $J 7.7,1.73), 7.28-7.32(6 \mathrm{H}, \mathrm{m}), 7.36$ (1 H, td, $J 7.4,1.2), 7.45-7.49(4 \mathrm{H}, \mathrm{m})$, $7.83(1 \mathrm{H}, \mathrm{dd}, J 7.9,1.7), 7.96(1 \mathrm{H}, \mathrm{dd}, J 8.2,1.2) ;{ }^{13} \mathrm{C} \mathrm{NMR}\left(\delta, 67 \mathrm{MHz}, \mathrm{CDCl}_{3}\right) 21.7,62.4$, $85.4,87.3,94.3,122.4,128.1,128.5,128.8,131.2,132.0,132.9,134.9,141.4,165.5$; highresolution MS (m/z, ES) found $\mathrm{MNH}_{4}{ }^{+}$368.1643. $\mathrm{C}_{25} \mathrm{H}_{22} \mathrm{NO}_{2}$ requires 368.1643.

8b: Use of $7 \mathbf{b}(2.0 \mathrm{~g}, 4.94 \mathrm{mmol})$ gave $\mathbf{8 b}$ as a brown oil $\left(1.83 \mathrm{~g}, 98 \%\right.$ ). IR (thin film) $v_{\max }$ $1733 \mathrm{~cm}^{-1} ;{ }^{1} \mathrm{H}$ NMR $\left(\delta, 270 \mathrm{MHz}, \mathrm{CDCl}_{3}\right) 1.10(3 \mathrm{H}, \mathrm{d}, J 6.9,1.13(3 \mathrm{H}, \mathrm{d}, J 6.7), 2.29(1 \mathrm{H}$, octet, $J$ 6.7), 5.83 (1 H, d, $J$ 5.4), 7.27 - 7.67 (13 H, m), $8.05(1 \mathrm{H}, \mathrm{d}, J 6.4) ;{ }^{13} \mathrm{C} \mathrm{NMR}(\delta, 67$ $\left.\mathrm{MHz}, \mathrm{CDCl}_{3}\right)$ 18.0, 18.7, 33.0, 70.4, 85.6, 86.3, 88.5, 94.8, 122.6, 123.5, 124.1, 125.4, 128.1, $128.4,128.6,130.3,130.8,131.8,131.9,132.0,134.3,165.3$; high-resolutions $\mathrm{MS}(\mathrm{m} / \mathrm{z}, \mathrm{ES})$ found $\mathrm{MH}^{+} 379.1692 ; \mathrm{C}_{27} \mathrm{H}_{23} \mathrm{O}_{2}$ requires 379.1693 .

\section{Synthesis of $(S)-4 b$.}

Using the procedure previously reported, ${ }^{1}$ and utilising (-)- $N$-methyl-ephedrine as the source of chirality, 2-hydroxy-2-methyl-3-butyne $(2.56 \mathrm{~mL}, 26.4 \mathrm{mmol})$ was transformed into $(S)$ - 
benzoic acid 4-hydroxy-1-isopropyl-4-methyl-pent-2-ynyl ester, isolated as a colourless oil ( $3.11 \mathrm{~g}, 45 \%$ ). The enantiomeric excess of this was determined as $72 \%$ by HPLC analysis of the corresponding 3,5-dinitrobenzoate ester (Chiralcel OD-H, 15\%, $i$-PrOH in hexane, 254 $\mathrm{nm}, 1 \mathrm{~mL} \min ^{-1}$ ), $t_{\mathrm{r}} 110.1$ (minor); 120.8 (major) [lit. ${ }^{1} t_{\mathrm{r}} 43.4$ (minor), 53.9. (major)]. Subsequent pyrolysis, also as previously described, ${ }^{1}$ resulted in the isolation of $(S)$-benzoic acid 1-isopropyl-prop-2-ynyl ester as a colourless oil (1.73 g, $72 \%)$.

Benzoic acid 1-isopropyl-3-phenyl-prop-2-ynyl ester.

(S)-Benzoic acid 1-isopropyl-prop-2-ynyl ester (1.73 g, $8.6 \mathrm{mmol})$ was stirred in dry triethylamine $(10 \mathrm{~mL})$, with copper(I) iodide (10 mol\%), $\mathrm{PdCl}_{2}\left(\mathrm{PPh}_{3}\right)_{2}(3 \mathrm{~mol} \%)$, and iodobenzene $(0.88 \mathrm{~mL}, 7.9 \mathrm{mmol})$. Light was excluded from the reaction and the mixture was left to stir for 2 days at room temperature. After addition to dichloromethane $(15 \mathrm{~mL})$ and saturated aqueous ammonium chloride $(10 \mathrm{~mL})$, the organic layer was separated, dried $\left(\mathrm{MgSO}_{4}\right)$, filtered and the solvent removed in vacuo. Column chromatography $(30 \%$ EtOAc/petroleum ether) gave (S)-benzoic acid 1-isopropyl-3-phenyl-prop-2-ynyl ester was as a pale yellow oil (2.19 g, $100 \%) .{ }^{1} \mathrm{H} \operatorname{NMR}\left(\delta, 270 \mathrm{MHz}, \mathrm{CDCl}_{3}\right) 1.11(3 \mathrm{H}, \mathrm{d}, J 6.9), 1.14$ (3 H, d, J 6.9), 1.98 - $2.10(1 \mathrm{H}, \mathrm{m}), 5.11(1 \mathrm{H}, \mathrm{d}, J 5.4), 7.10$ - $7.13(3 \mathrm{H}, \mathrm{m}), 7.30$ - 7.33 (3 H, m), 7.47 - $7.51(1 \mathrm{H}, \mathrm{m}), 7.70-7.74(3 \mathrm{H}, \mathrm{m}) ;{ }^{13} \mathrm{C} \mathrm{NMR}\left(\delta, 270 \mathrm{MHz}, \mathrm{CDCl}_{3}\right)$ 17.6, 18.2, $32.7,69.1,85.6,94.5,127.2,128.2,128.3,129.5,130.1,131.7,132.8,137.2,164.7$.

(S)-4-Methyl-1-phenyl-pent-1-yn-3-ol

$(S)$-Benzoic acid 1-isopropyl-3-phenyl-prop-2-ynyl ester (2.19 g, $7.9 \mathrm{mmol})$ was stirred in methanol $(10 \mathrm{~mL})$ and aqueous $1 \mathrm{M} \mathrm{NaOH}(10 \mathrm{~mL})$ for $30 \mathrm{~min}$. To this was added $\mathrm{H}_{2} \mathrm{O}(20$ $\mathrm{mL})$ and the mixture extracted with dichloromethane $(3 \times 10 \mathrm{~mL})$. The combined organic layers were dried $\left(\mathrm{MgSO}_{4}\right)$, filtered and the solvent removed in vacuo. Column chromatography (10\% EtOAc/petroleum ether) gave $(S)$-4-methyl-1-phenyl-pent-1-yn-3-ol $[(S)-\mathbf{4 b}]$ as a pale yellow oil $(1.30 \mathrm{~g}, 95 \%)$. 
Synthesis of $(S)-8 b$ and subsequent reaction with $\mathrm{CpCo}(\mathrm{CO})_{2}$.

Using the procedures described above, $(S)-4 \mathbf{b}(0.250 \mathrm{~g}, 1.43 \mathrm{mmol})$ was first converted into $(S)-7 \mathbf{b}(0.551 \mathrm{~g}, 95 \%)$, and this in turn lead to the formation of $(S)-8 \mathbf{b}(0.25 \mathrm{~g}, 48 \%)$. Subsequent reaction of $(S)-8 \mathbf{b}(0.100 \mathrm{~g}, 0.26 \mathrm{mmol})$ with $\mathrm{CoCp}(\mathrm{CO})_{2}$ using conditions B as described above, lead to the isolation of scalemic metallocenes as a 3.8:1 ratio of $\mathbf{1 1 b}$ and 12b, isolated as a red solid $(0.053 \mathrm{~g}, 38 \%)$. $[\alpha]_{589}^{24.5}=-172.0$. Enantiomeric excess of $\mathbf{1 1 b}(74 \%$ e.e.) and 12b (72\% e.e.) determined by HPLC analysis (Chiralcel OD-H, $4 \% i$-PrOH in

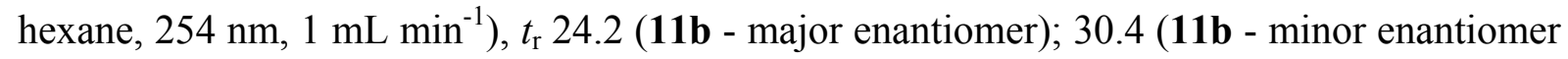
$+\mathbf{1 2 b}$ - minor enantiomer), 44.3 (12b - major enantiomer). 


\section{X-Ray crystallography - Compound 9a}

Data were collected at $120 \mathrm{~K}$ using a Nonius Kappa CCD area detector diffractometer mounted at the window of molybdenum rotating anode $(50 \mathrm{KV}, 90 \mathrm{~mA}, \lambda=0.71069 \AA)$. The crystal-to-detector distance was $30 \mathrm{~mm}$ and $\phi$ and $\Omega$ scans $\left(2.0^{\circ}\right.$ increments, $12 \mathrm{~s}$ exposure time) were carried out to fill the Ewald sphere. Data collection and processing were carried out using the COLLECT, ${ }^{2}$ DENZO $^{3}$ and maXus $^{4}$ and empirical absorption correction was applied using SADABS. ${ }^{5}$ The structure was solved by heavy-atom method using DIRDIF99 ${ }^{6}$ and refined anisotropically( non-hydrogen atoms) (except for solvent molecule) by full-matrix least-squares on $F^{2}$ using the SHELXL- $97^{7}$ program. The $\mathrm{H}$ atoms were calculated geometrically and refined with riding model. Crystal data and refinement details are given below.

Crystal data 9a. $\mathrm{C}_{25} \mathrm{H}_{21} \mathrm{CoO}_{2} \cdot 1 / 6 \mathrm{CH}_{3} \mathrm{OH}, M=417.69$, Trigonal, $\mathrm{a}=33.155(5), \mathrm{b}=$ $33.155(5), \mathrm{c}=9.4226(7) \AA, \alpha=90.00, \beta=90.00, \gamma=120.00^{\circ}, V=8970(2) \AA^{3}$, Space group $\mathrm{R} 3, Z=18, D_{\mathrm{c}}=1.392 \mathrm{Mg} / \mathrm{m}^{3}, \mu=0.880 \mathrm{~mm}^{-1}$, reflections measured 13654, reflections unique 3277 with Rint $=0.0826, T=120(2) K$, Final $\mathrm{R}$ indices $\{\mathrm{I}>2 \sigma(I)] \mathrm{R} 1=0.0630$, $\mathrm{wR} 2=0.1483$ and for all data $\mathrm{R} 1=0.1380, \mathrm{wR} 2=0.1829$. 
Representation of 9a with numbering scheme.

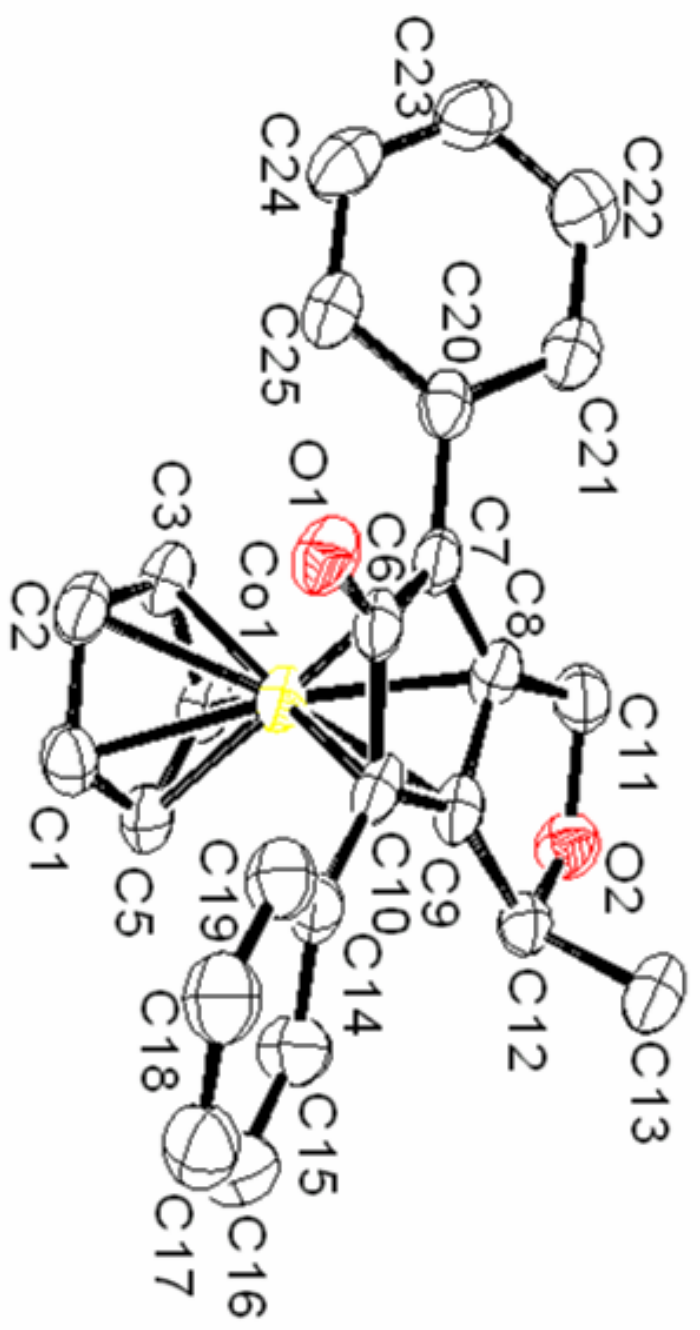




\section{X-Ray crystallography - Compound 11a}

Data were collected at $120 \mathrm{~K}$ using a Bruker SMART APEX2, CCD area detector diffractometer mounted at the window of Synchrotron radiation (Daresbury SRC station 9.8) with $\lambda=0.0 .6902 \AA) .{ }^{8,9}$ Data collection, cell refinement and processing were carried out using the APEX2, ${ }^{10}$ SAINT $^{11}$ and empirical absorption correction was applied using SADABS. ${ }^{12}$ The structure was solved by heavy- atom method using DIRDIF99 ${ }^{6}$ and refined anisotropically( non-hydrogen atoms) (except for solvent molecule) by full-matrix leastsquares on $F^{2}$ using the SHELXL- $97^{7}$ program. The $\mathrm{H}$ atoms were calculated geometrically and refined with riding model. Crystal data and refinement details are given below.

Crystal data 11a. $\mathrm{C}_{31} \mathrm{H}_{23} \mathrm{CoO}_{3}, M=502.42$, Triclinic, $\mathrm{a}=10.512(3), \mathrm{b}=10.734(3), \mathrm{c}=$ 11.473(3) $\AA, \alpha=68.257(3), \beta=71.825(3), \gamma=81.176(4)^{\circ}, V=1141.4(5) \AA^{3}$, Space group P1, $Z=2, D_{\mathrm{c}}=1.462 \mathrm{Mg} / \mathrm{m}^{3}, \mu=0.785 \mathrm{~mm}^{-1}$, reflections measured 8280 , reflections unique 3868 with Rint $=0.0674, T=120(2) K, \quad$ Final $\mathrm{R}$ indices $\{\mathrm{I}>2 \sigma(I)] \mathrm{R} 1=0.0537, \mathrm{wR} 2=0.1146$ and for all data $\mathrm{R} 1=0.1060, \mathrm{wR} 2=0.1375$. 
Representation of 11a with numbering scheme.

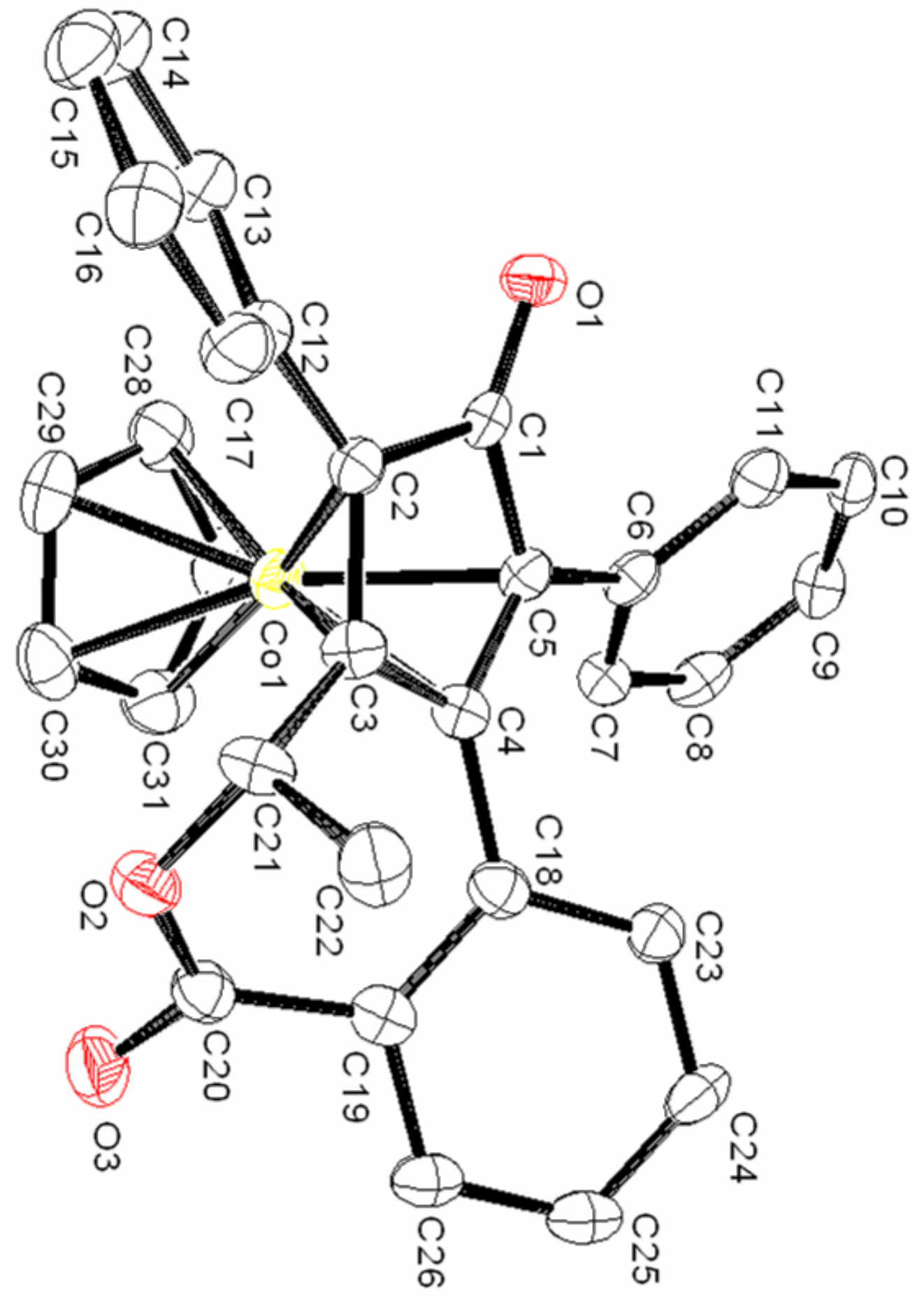




\section{References}

(1) Boyall, D.; López, F.; Sasaki, H.; Frantz, D.; Carreira, E. M. Org. Lett., 2000, 2, 4233.

(2) Collect Data collection and processing user interface: Collect: Data collection software, R. Hooft, Nonius B.V., 1998.

(3) Denzo Data collection and processing software: Z. Otwinowski and W. Minor, Methods in Enzymology, Volume 276: Macromolecular Crystallography, part A, p.307-326, 1997, C.W. Carter, Jr and R.M. Sweet, Eds., Academic Press.

(4) maXus solution and refinement software suite: S. Mackay, C.J. Gilmore, C. Edwards, M. Tremayne, N. Stewart, K. Shankland. maXus: a computer program for the solution and refinement of crystal structures from diffraction data .

(5) Sheldrick, G. M.(2003). SADABS - Bruker Nonius area detector scaling and absorption correction - V2.10.

(6) DIRDIF99 program system. P. T. Beurskens, G. Beurskens, W. P. Bosman, R. de Gelder, S. Garcia-Granda, R. O. Gould, R. Israel and J. M. M. Smits, Crystallography Laboratory, University of Nijmegen, The Netherlands. 1999.

(7) SHELX97 [Includes SHELXS97, SHELXL97, CIFTAB (and SHELXA) ] - Programs for Crystal Structure Analysis (Release 97-2). G. M. Sheldrick, Institüt für Anorganische Chemie der Universität, Tammanstrasse 4, D-3400 Göttingen, Germany, 1998.

(8) Station 9.8: Cernik, R. J.; Clegg, W.; Catlow, C. R. A.; Bushnell-Wye, G.; Flaherty, J. V.; Greaves, G. N.; Hamichi, M.; Burrows, I.; Taylor. D. J.; Teat, S. J. J. Synchotron Radiation, 1997, 4, 279.

(9) Synchrotron chemical crystallography: Clegg, W.; J. Chem. Soc., Dalton Trans. 2000, 3223.

(10) Data collection: APEX2, Bruker AXS Inc., Madison, USA, 2004. 
(11) Data reduction (frames integration): SAINT, Bruker AXS Inc., Madison, USA, 2004.

(12) Absorption correction: SADABS, Bruker AXS Inc., Madison, USA, 2004. 